\title{
QUANTUM PHASE SHIFT IN CHERN-SIMONS MODIFIED GRAVITY
}

\author{
K.K. Nandi ${ }^{1,2,3, a}$, I.R. Kizirgulov ${ }^{3, b}$, O.V. Mikolaychuk ${ }^{3, c}$, N.P. \\ Mikolaychuk $^{3, d}$, A.A. Potapov ${ }^{3, e}$ \\ ${ }^{1}$ Department of Mathematics, University of North Bengal, Siliguri 734013, \\ India \\ ${ }^{2}$ Joint Research Laboratory, Bashkir State Pedagogical University, Ufa 450000, \\ Russia \\ ${ }^{3}$ Department of Theoretical Physics, Sterlitamak State Pedagogical Academy, \\ Sterlitamak 453103, Russia \\ ${ }^{a}$ Email: kamalnandi1952@yahoo.co.in \\ ${ }^{b}$ Email: kizirgulovir@mail.ru \\ ${ }^{c}$ Email: lacertida@mail.ru \\ ${ }^{d}$ Email: zetavoznichego@mail.ru \\ ${ }^{e}$ Email: potapovaa2008@rambler.ru
}

\begin{abstract}
Using a unified approach of optical-mechanical analogy in a semiclassical formula, we evaluate the effect of Chern-Simons modified gravity on the quantum phase shift of de Broglie waves in neutron interferometry. The phase shift calculated here reveals, in a single equation, a combination of effects coming from Newtonian gravity, inertial forces, Schwarzschild and Chern-Simons modified gravity. However the last two effects, though new, turn out to be too tiny to be observed, and hence only of academic interest at present. The approximations, wherever used, as well as the drawbacks of the non-dynamical approach are clearly indicated.
\end{abstract}

PACS numbers(s): 95.85.Ry, 12.15.Ff, 04.50.+h

\section{Introduction}

Investigations at a semiclassical level of gravitational effects on quantum interference are expected to be useful for the development of a theory of quantum gravity [1]. Although the full theory is yet to appear, outcomes of early seminal experiments by Colella, Overhauser and Werner (COW) [2] and an improved later experiment by Werner, Staudenmann and Colella (WSC) [3], measuring respectively the effects of Newtonian gravity and Coriolis force on the phase shift of neutrons, continue to provide essential resources in this direction. The effect of Coriolis force was first suggested by Page [4]. Several other related works have also contributed useful information. For instance, a 
semiclassical treatment reveals that quantum uncertainty in the source variables induces uncertainties in the metric components of gravity in a specific manner [5]. Another important phenomenon is the neutrino flavor oscillation induced by gravity [6-9]. A different kind of theoretical approach in the calculations of the phase of quantum particles, neutrinos included, has yielded a very interesting result: The Dirac spin $1 / 2$ particle has the exact covariant Stodolsky [10] phase $S / \hbar=(1 / \hbar) \int p_{\mu} d x^{\mu}$ in a static gravity field [11]. Some of these investigations could provide appropriate theoretical backgrounds for astrophysical applications, e.g., in the atmospheric neutrino experiments [12], or in the observations involving $\gamma$-ray bursts [13].

On the other hand, the developments in matterwave interferometry have shown a greater promise (over photon interferometry) in the measurements of the phase shift due to Earth's gravity and axial rotation. In the absence of external forces, the interference with itself of the de Broglie wave associated with an ensemble of particles allows us to predict, via Huygen's principle, the motion of the wave. The presence of an external field modifies the motion causing a shift in the interference fringes. A classic example is the AharonovBohm (AB) fringe shift which provides information as to how the motion of electrons are modified in the presence of a magnetic potential. However, the situation becomes more complicated when one introduces the effects of gravity and rotation on matterwave interferometry.

An intuitive and elegant special relativistic treatment of the quantum phase shift for thermal neutrons has been proposed by Anandan [14]. However, the effects of gravity and rotation are still considered as separate components. On the other hand, a more comprehensive analysis involving gravity and inertial forces would require the use of complete geometrical framework of curved spacetime. The special relativistic effects should follow only as a limiting case. Anandan's semiclassical treatment paves the way for the application of simpler "Newtonian" form of equations of optical-mechanical analogy in curved spacetime [1519]. These equations provide a substitute for geometrical framework though only for calculational purposes. Using them, the corrections to the phase shift had been worked out [20] in certain spacetimes: A "Coriolis" force leading to a gravitational analog of the quantum $\mathrm{AB}$ effect appeared naturally from the application of a "Lorentz force" form of the geodesic equation in Kerr and KerrSen [21] spacetime. (Optical analogs are artifacts designated by ".." and they can be easily translated into actual mechanical quantities, see below.)

A different genre of theory that leads to rotating solution different from that of Kerr is Chern-Simons (hereafter CS) modified gravity. A reasonable summary of the timeline and study of CS modified gravity is in order. The CS modified gravity descends from all versions of string theory upon 4-dimensional compactification due to the Green-Schwarz anomaly-cancelling mechanism [21,22]. The first group to study CS modified gravity as a string inspired correction to general relativity consisted of Alexander, Peshkin and Sheikh-Jabbari [23] as well as Alexander and Gates [24]. The initial non-dynamical formulation of CS modified gravity was presented by Jackiw and Pi [25] who also showed that the Schwarzschild metric as well as a special type of gravitational wave is a solution 
of CS modified gravity. Guarrera and Hariton [26] showed, using Jackiw-Pi CS coupling field, that Reissner-Nordström is also a solution of CS gravity. The first spinning black hole solutions were found by Alexander and Yunes [27, 28]. These solutions represent spinning black holes in the far field limit but are not axisymmetric. Smith, Erickcek, Caldwell, and Kamionkowski [29] evaluated constraints on the CS parameter space from current satellite experiments such as LAGEOS and Gravity Probe-B. Their approach can be regarded as an extension of the Jackiw-Pi formulation because the action contains a scalar field that is time varying but spatially homogeneous. Grumiller and Yunes [30] performed a detailed study of exact solutions that could represent spinning black holes with arbitrary CS coupling fields. This is the most complete search for black hole-like solutions currently available in CS modified gravity for a non-dynamical field. Alexander and Yunes [31] studied the coupling of fermions to CS modified gravity. Yunes and Sopuerta [32] studied perturbations of Schwarzschild black holes in CS modified gravity.

Konno, Matsuyama, Asano and Tanda [33] (hereafter KMAT) studied a solution for a slowly rotating black hole in CS modified gravity derived previously by Konno, Matsuyama and Tanda [34] (hereafter KMT). The KMT slow rotating solution corresponds to a (special) spatial coupling field. The solution differs from all others in that it is axisymmetric, among other things. However, the solution and the non-dynamical approach have several unphysical features. The solution is based on a purely ad hoc non-dynamical scalar, which plagues the well-posedness of the initial value problem [32]. Moreover, the solution is nonunique and does not carry over to the dynamical sector because the scalar field has infinite energy, which in turn prevents a self-consistent perturbation of the general relativity solutions [35]. Despite these drawbacks, the Alexander-Yunes far field solution and the KMT slow rotating solution are useful for understanding the CS effects on physical observables. KMAT [33] showed that their solution remarkably explains galactic flat rotation curves without the need to invoke hypothetical dark matter. They also calculated the effect of frame dragging on spin precession, which could provide a way to observationally test CS gravity.

In this paper, we shall consider the KMT solution [33,34] to study the effects of CS modified gravity on the phase shift in neutron interferometry using, in addition, the equations of optical-mechanical analogy for rotating sources [36]. The final formula for phase shift shows a combination of different effects. We also recover, as a limiting case, the observed zeroth order effects of Newtonian gravity and Coriolis force. As shown by KMAT, one of the CS constants appear as constant tangential velocity in the galactic halo. Thus one might treat its appearance in the phase shift as a non-local effect similar to the effect of, say, cosmological constant in local experiments. Our calculation shows that the effect of CS constants, which represents dragging of inertial frames, appear only in the second order of slow rotation parameter and thus too tiny.

The paper is organized as follows: To be reasonably informative, we briefly state in Sec.II the CS action together with the KMT solution and discuss stability of circular orbits, which is of crucial importance. The involved approximations on the geodesic equation are clearly mentioned. Sec.III describes the 
essence of Anandan's argument which provides a core element of the ensuing analysis. To familiarize the readers at large with what we refer to as optical mechanical analogy in curved spacetime, we briefly review its contents in Sec.IV. We calculate the exact phase shift in CS gravity in Sec.V and extract the corrections in Sec.VI. In Sec.VII, we summarize the results of the paper together with a brief review of the difficulties of the KMT solution and the non-dynamical approach. We shall take $G=c_{0}=1$, unless specifically displayed.

\section{The CS action, KMT solution and stability}

(a) CS modified gravity follows from the action [33,34]

$$
I=\frac{1}{16 \pi} \int d^{4} x\left(\sqrt{-g} R-\frac{1}{2} v_{\mu} K^{\mu}\right),
$$

where $v_{\mu} \equiv \partial_{\mu} \vartheta$ is an external 4-vector, the so-called the embedding coordinate. The CS topological current $K^{\mu}$ is given by

$$
K^{\mu}=\varepsilon^{\mu \alpha \beta \gamma}\left[\Gamma_{\alpha \tau}^{\sigma} \partial_{\beta} \Gamma_{\gamma \sigma}^{\tau}+\frac{2}{3} \Gamma_{\alpha \tau}^{\sigma} \Gamma_{\beta \eta}^{\tau} \Gamma_{\gamma \sigma}^{\eta}\right] .
$$

where $\varepsilon^{\sigma \mu \alpha \beta}$ the Levi-Civita tensor density of weight one. Variation of the action with respect to the metric $g_{\mu \nu}$ leads to the CS field equation

$$
G^{\mu \nu}+C^{\mu \nu}=-8 \pi T^{\mu \nu},
$$

where $G^{\mu \nu} \equiv R^{\mu \nu}-\frac{1}{2} g^{\mu \nu} R$ is the Einstein tensor, $T^{\mu \nu}$ is the usual energymomentum tensor, and $C^{\mu \nu}$ is the Cotton tensor given by

$C^{\mu \nu}=-\frac{1}{2 \sqrt{-g}}\left[v_{\sigma}\left(\varepsilon^{\sigma \mu \alpha \beta} \nabla_{\alpha} R_{\beta}^{\nu}+\varepsilon^{\sigma \nu \alpha \beta} \nabla_{\alpha} R_{\beta}^{\mu}\right)+\left(\nabla_{\sigma} v_{\tau}\right)\left({ }^{*} R^{\tau \mu \sigma \nu}+{ }^{*} R^{\tau \nu \sigma \mu}\right)\right]$

In the above, the dual Riemann tensor is defined by ${ }^{*} R_{\sigma}^{\tau}{ }^{\mu \nu} \equiv \frac{1}{2} \varepsilon^{\mu \nu \alpha \beta} R_{\sigma \alpha \beta}^{\tau}$ and $v_{\tau \sigma} \equiv \nabla_{\sigma} v_{\tau}=\partial_{\sigma} \partial_{\tau} \vartheta-\Gamma_{\tau \sigma}^{\lambda} \partial_{\lambda} \vartheta$ is a symmetric tensor. The Bianchi identity $\nabla_{\mu} G^{\mu \nu}=0$ and the equation of motion $\nabla_{\mu} T^{\mu \nu}=0$ together imposes the condition $[33,34]$

$$
0=\nabla_{\mu} C^{\mu \nu}=\frac{1}{8 \sqrt{-g}} v^{\nu *} R_{\tau}^{\sigma \mu \lambda} R_{\sigma \mu \lambda}^{\tau} .
$$

(b) KMAT [33] consider perturbation around the Schwarzschild solution of mass $M$ in the small expansion parameter $\epsilon(\equiv J / M r)$ where $J$ is the angular momentum. Then, for a slowly rotating star, the KMT solution [34] in the case of a spacelike vector $v_{\mu}=\partial_{\mu} \vartheta=\partial_{\mu} z=\partial_{\mu}(r \cos \theta)=(0, \cos \theta,-r \sin \theta, 0)$ becomes

$d s^{2}=-\left(1-\frac{2 M}{r}\right) d t^{2}+\left(1-\frac{2 M}{r}\right)^{-1} d r^{2}+r^{2}\left(d \theta^{2}+\sin ^{2} \theta d \phi^{2}\right)-2 r^{2} \omega(r) d t d \phi$, 
the $\theta$-dependence of the $(t \phi)$-component is restricted by Eq. (5), and

$$
\omega(r)=\frac{C_{1}}{r^{2}}\left(1-\frac{2 M}{r}\right)+\frac{C_{2}}{r^{3}}\left[r^{2}-2 M r-4 M^{2}+4 M(r-2 M) \ln (r-2 M)\right]
$$

where $C_{1}$ and $C_{2}$ are constants (hereafter called CS constants) characterizing slow rotation i.e., $C_{1}, C_{2} \sim O(\epsilon)$. From the metric, it is clear that $\omega \sim$ (length) ${ }^{-1}$, which implies that $C_{1} \sim$ (length) and $C_{2}$ is truly dimensionless.

We should clearly mention that we are considering only the non-dynamical formulation of CS modified gravity, where the CS scalar field in the KMT solution satisfies the evolution equation only to leading order in the spin [35] (See Ref. [29] for the dynamical formulation). However, it is well to remember that the non-dynamical theory have several physical drawbacks as mentioned in the introduction. The solution (6) is based on the choice of a particular non-dynamical scalar defined by $\vartheta=r \cos \theta / \lambda_{0}$, where $\lambda_{0}$ is a constant. On the other hand, in the non-dynamical framework, there are no well-motivated physical reasons for particular choices of the scalar field except for simplifying the equations. Furthermore, for a given choice of CS scalar the Pontryagin constraint significantly restricts the class of allowed solutions, even to the point where the non-dynamical theory may be over-constrained and lack a well-posed initial value problem [32]. The hope to imbed the KMT solution in the dynamical sector is dashed because the stress energy associated with $\vartheta=r \cos \theta / \lambda_{0}$ is infinite. As shown by Yunes and Pretorius [35], even a more general choice of the KMT scalar does not heal the disease. Therefore the KMT metric is not a self-consistent solution to the dynamical field equations. Yunes and Pretorius [35] have found a new stationary axisymmetric solution for a different non-dynamical scalar. Although the canonical $\vartheta$ is not allowed by this family of solutions, it does bypass the problem with the stress tensor and is compatible with the dynamical framework. The existence of new independent solution shows that a solution in the non-dynamical theory such as that of KMT can not be a unique black hole solution.

In the CS modified theory, as well as in general relativity, the standard geodesic description holds if and only if the back-reaction is neglected in the equations of motion. Such an approximation is valid, for example, in the extreme-mass ratio limit. It is not valid, for instance, in the strong field of equal mass binaries. Only under the assumption that the effect of test particle's self-force on its own trajectory be neglected, the geodesic calculations lead to the transverse circular velocity $v^{\phi}$ in the equatorial plane $(\theta=\pi / 2)$ as

$$
v^{\phi}=r \frac{d \phi}{d t}= \pm \sqrt{\frac{M}{r}}+\left[r \omega(r)+\frac{r^{2}}{2} \omega^{\prime}(r)\right]
$$

where $\omega^{\prime}=d \omega / d r$. The first term clearly comes from the Schwarzschild metric while the second term becomes a constant at large $r$, i.e.,

$$
v^{\phi} \simeq \pm \sqrt{\frac{M}{r}}+\frac{C_{2}}{2}
$$


This result remarkably explains the observed flat rotation curves in the galactic halo. Essentially $C_{2}$ is responsible for frame dragging that decays as $r^{-4}$.

(c) It is however not a priori evident if the circular orbits in the spacetime are stable. On the other hand, stability of circular orbits in the galactic halo is a crucial requirement if any model of the halo has to be physically viable. We have carried out the necessary calculations with the potential

$$
V(r)=-\left(1-\frac{2 M}{r}\right)\left(1+\frac{L^{2}}{r^{2}}\right)-2 E L \omega(r),
$$

where the constants $E$ and $L$ are respectively the conserved relativistic energy and angular momentum per unit rest mass of the test particle in a circular orbit. Putting in the relevant expressions [33] for $E$ and $L$ in $\left.\frac{d^{2} V}{d r^{2}}\right|_{r=R}$, we find the following results: The expression for $V^{\prime \prime}=\left.\frac{d^{2} V}{d r^{2}}\right|_{r=R}$ at any arbitrary but fixed radius $r=R$ depends only on $C_{1}$ and $C_{2}$, and that $\left.\frac{d^{2} V}{d r^{2}}\right|_{r=R}<0$ only for values of these constants very small relative to the length scale $M$, for instance $C_{1}, C_{2} \in[0.001,0.0002]$ (see Fig.1 for details), which of course is in perfect accordance with the slow rotation approximation. So we conclude that the orbits are indeed stable for very small values of $C_{1}, C_{2}$. Otherwise, the orbits are unstable.

\section{Special relativistic phase shift: Anandan's formula}

Before we develop the equations for CS gravity, it is useful to have a brief preview of the special relativistic phase shift because it will ultimately provide the limiting case. (All special relativistic quantities will be denoted by tilde). Using intuitive arguments, Anandan [14] proposed a general formula for the quantum phase shift based on a correspondence between the shift and the classical special relativistic equations of motion. Consider two de Broglie wavelets originating at $\mathrm{A}$ and interfering at $\mathrm{B}$, one travelling along $\mathrm{ADB}$ and the other along $\mathrm{ACB}$, the plane $\mathrm{ADBC}$ being rotated by $180^{\circ}$ about the horizontal axis AO (Fig.2) while the Earth itself is rotating about its axis with angular velocity $\vec{\Omega}$. The vertical direction is determined by the resultant of gravity and the centrifugal force of Earth. In the absence of any external forces, $\mathrm{BD}=\mathrm{BC}$ and we take $\mathrm{CD}=d$, and $\mathrm{AB}=l$. The external fields cause a shift in the angular position of $\mathrm{B}$ by $\delta \theta \neq 0$ and thereby cause a phase shift. Let $\tilde{v}$ be the velocity of the classical particle and $\delta \tilde{v}$ be the change in the velocity in the plane of interference. Since $\delta \theta$ is small, $\sin \delta \theta \simeq \delta \theta \simeq \frac{\delta \tilde{v}}{\tilde{v}}$. Then the special relativistic phase shift $\Delta \phi_{S R}$ can be calculated as [14]:

$$
\Delta \phi_{S R}=\tilde{\kappa} d \frac{\delta \tilde{v}}{\tilde{v}}=\frac{\tilde{\kappa} d}{\tilde{v}} \frac{d \tilde{v}_{\perp}}{d t} \frac{l}{\tilde{v}}=\frac{\tilde{p} A}{\hbar \tilde{v}^{2}} \frac{d \tilde{v}_{\perp}}{d t}=\frac{\tilde{\gamma} m A d \tilde{v}_{\perp} / d t}{\tilde{v} \hbar},
$$

where $\tilde{\gamma}=\left(1-\tilde{v}^{2} / c_{0}^{2}\right)^{-1 / 2}, A=l d$ is the planar area (assumed small) enclosed by the two paths of interfering beams, $m$ is the neutron mass, $\tilde{p}(=\hbar \tilde{\kappa})$ is the momentum in flat space, and $d \tilde{v}_{\perp} / d t$ is the component of acceleration in the 
vertical direction CD. This remarkable general formula, Eq.(11), at once gives rise to several individual components.

The following cases were considered: (i) A particle with charge $e$ moving in a magnetic field $B$. Then, $\tilde{\gamma} d \tilde{v}_{\perp} / d t=e B \tilde{v} / m$, which, when used in Eq.(11), immediately yields the Aharonov-Bohm (AB) effect, viz.,

$$
\Delta \widetilde{\phi}_{A B}=e B A / \hbar \text {. }
$$

(ii) A spinless particle of mass $m$ in a gravitational field, $d \tilde{v}_{\perp} / d t=-g$, where $g$ is the gravitational acceleration on the surface of the Earth. Using the expression for the relativistic momentum $\tilde{\gamma}^{2}=1+\tilde{p}^{2} / m^{2} c_{0}^{2}$ and the de Broglie relation $\tilde{p}=\hbar \tilde{\kappa}$, Eq.(11) leads to the following result:

$$
\Delta \widetilde{\phi}_{g}=-g A m^{2} / \hbar^{2} \tilde{\kappa}-g A \tilde{\kappa} / c_{0}^{2} .
$$

The COW experiment was accurate enough to measure the first quantum term, but not the second, that is, the so called relativistic term. (iii) A particle moving in the Coriolis force field of Earth so that, to first order in $\Omega, d \tilde{v}_{\perp} / d t=$ $2|\vec{\Omega} \times \overrightarrow{\tilde{v}}|=2 \Omega_{n} \tilde{v}$, where $\Omega_{n}$ is the component of Earth's angular velocity $\vec{\Omega}$ normal to the apparatus. Using the Planck-Einstein law $E / c_{0}=\hbar \tilde{\omega}=m c_{0} \tilde{\gamma}$, one finds from Eq.(11) that

$$
\Delta \widetilde{\phi}_{c o r}=\frac{2 \tilde{\omega} A \Omega_{n}}{c_{0}}
$$

Using the dispersion relation

$$
\tilde{\omega}^{2}-\tilde{\kappa}^{2}=\frac{m^{2} c_{0}^{2}}{\hbar^{2}}
$$

we find from Eq.(14) that

$$
\Delta \widetilde{\phi}_{c o r} \simeq \frac{2 m A \Omega_{n}}{\hbar}+\frac{\hbar A \Omega_{n} \tilde{\kappa}^{2}}{m c_{0}^{2}}
$$

The WSC experiment has tested the first nonrelativistic term to within a good accuracy. (iv) This effect comes from the coupling of particle's spin to the background curvature. Again using Eq.(11) together with the Mathisson-Papapetrou force [37], the shift comes out to be

$$
\Delta \phi_{\text {s.c. }}=-\frac{\hbar G M A \tilde{\omega}}{m c_{0}^{3} R^{3}}
$$

where $G$ is the Newtonian gravitational constant, $M$ is the gravitating mass and $R$ is the distance from the center. (We shall not discuss this spin-curvature component in this article.) With all the above in view, we proceed to familiarize the readers with the salient features of our approach in the curved spacetime regime. 


\section{Optical-Mechanical equations in curved spacetime}

The central idea is to introduce an optical-mechanical analogy in curved spacetime. The exact optical form of geodesic equations in the spherically symmetric case of general relativity was originally derived in Ref.[15]. The equations provide an excellent tool that enables one to visualize the problems of geometrical optics as problems of classical mechanics and vice versa. The first step is to find out an effective optical refractive index $n$ that is formally equivalent to the geometrized gravity field. This step in itself is not new. Usually the index equivalent to the exterior Schwarzschild field is approximately taken to be $n \approx 1+2 M G / r c_{0}^{2}$, where $M$ is the central gravitating mass. We derive the exact expression as below.

Consider a static, spherically symmetric, but not necessarily vacuum, solution of general relativity written in isotropic coordinates

$$
d s^{2}=h(\vec{r}) c_{0}^{2} d t^{2}-\Phi^{-2}(\vec{r})|d \vec{r}|^{2},
$$

where $\vec{r} \equiv(x, y, z)$ or $(r, \theta, \varphi)$, and $h, \Phi$ could be the solution of Einstein's field equations. Many metrics of physical interest can be put into this isotropic form. The coordinate speed of light $c(\vec{r})$ is determined by the condition that the geodesic be null $\left(d s^{2}=0\right)$ :

$$
c(\vec{r})=\left|\frac{d \vec{r}}{d t}\right|=c_{0} \Phi \sqrt{h},
$$

which immediately provides an effective index of refraction for light in the gravitational field given by

$$
n(\vec{r})=\frac{1}{\Phi \sqrt{h}} .
$$

The concept of optical mechanical analogy can be used to recast the geodesic equation for both massive and massless particles into a single, exact Newtonian "F $=m \mathbf{a}$ " type of equation given by [16]

$$
\frac{d^{2} \vec{r}}{d A^{2}}=\vec{\nabla}\left(\frac{1}{2} N^{2} c_{0}^{2}\right), N(\vec{r})=n(\vec{r}) \sqrt{1-\frac{m^{2} c_{0}^{4} h}{E_{0}^{2}}}, d A=\frac{d t}{n^{2}},
$$

where $m$ is the rest mass of the particle, $E_{0}$ is the conserved total energy, $\vec{\nabla}$ is the gradient operator. All the standard geodesic equations in Schwarzschild gravity including some new insights in cosmology follow from the above equation. This remarkably simple feature of the geodesic equations is brought about by the use of the stepping parameter $A$, having dimension of length, first introduced by Evans and Rosenquist [19]. Eqs. (20) provide an easy way to introduce quantum relations so that the geodesic motion of a particle can be interpreted as motion of de Broglie matter waves in a dispersive medium with an effective index of refraction $N(\vec{r}, \lambda)=n(\vec{r}) / \sqrt{1+\left(\lambda / \lambda_{c}\right)^{2}}$ where $\lambda_{c}$ is the Compton wavelength given by $\lambda_{c}=2 \pi \hbar / m c_{0}$ and $\lambda=\tilde{\lambda} \Phi^{-1}$ is the physical wavelength measured 
in a gravity field. This interpretation allows us to extend in a straightforward manner the classical optical-mechanical analogy into the quantum regime.

Alsing [36] has subsequently extended the method to broader class of metrics in general relativity and his equation is going to provide the basic tool in what follows. Consider the most general form of the metric given by

$$
d s^{2}=g_{00} c_{0}^{2} d t^{2}+2 g_{0 i} c_{0} d t d x^{i}+g_{i j} d x^{i} d x^{j}, \quad i, j=1,2,3 .
$$

Define the proper time $d \tau$, proper length $d l$ and the velocity $v$ measured with respect to this proper time as

$$
\begin{gathered}
v^{i}=\frac{d x^{i}}{d \tau}, \\
\left.\frac{d s}{c_{0}}\right|_{v=0}=d \tau=\frac{\sqrt{h}}{c_{0}}\left(c_{0} d t-g_{i} d x^{i}\right), \quad g_{00}=h, \\
d l^{2}=\gamma_{i j} d x^{i} d x^{j}=\left(-g_{i j}+\frac{g_{0 i} g_{0 j}}{g_{00}}\right) d x^{i} d x^{j}, \quad v^{2}=\gamma_{i j} v^{i} v^{j}, \\
g_{i}=-\frac{g_{0 i}}{g_{00}}, \quad g^{i}=\gamma^{i j} g_{j}=-g^{0 i} .
\end{gathered}
$$

The angular momentum $g_{i}$ and the proper velocity $v_{i}$ are vectors defined in the 3-space characterized by the metric $\gamma_{i j}$, which is used to raise or lower the indices of these 3 -vectors. Now, metric (21) can be rewritten as

$$
d s^{2}=h\left(c_{0} d t-g_{i} d x^{i}\right)^{2}\left(1-v^{2} / c_{0}^{2}\right) .
$$

and the conserved energy $E$ is given by [38]

$$
E=m c_{0}^{2} g_{0 \alpha} \frac{d x^{\alpha}}{d s}=\frac{m c_{0}^{2} \sqrt{h}}{\sqrt{1-v^{2} / c_{0}^{2}}}, \quad \alpha=0,1,2,3 .
$$

The variational principle for the geodesics following from Eq.(21) is given by

$$
\delta \int_{\vec{x}_{1}, t_{1}}^{\vec{x}_{2}, t_{2}} m c_{0} d s=\delta \int_{\vec{x}_{1}, t_{1}}^{\vec{x}_{2}, t_{2}} L d t=\delta \int_{\vec{x}_{1}, t_{1}}^{\vec{x}_{2}, t_{2}} m c_{0}^{2} \sqrt{h(\vec{r})} \sqrt{1-v^{2}(\vec{r}, \tilde{v}) / c_{0}^{2}} \tilde{\beta}(\vec{r}, \tilde{v}) d t=0
$$

where

$$
\tilde{\beta}=1-\frac{g_{i} \tilde{v}^{i}}{c_{0}}, \quad \tilde{v}^{i}=\frac{d x^{i}}{d t}, \quad v^{i}=\frac{\tilde{v}^{i}}{\tilde{\beta} \sqrt{h}} .
$$

From the Lagrangian $L$, let us find the momenta conjugate to $\tilde{v}^{i}$. (Flat space quantities are designated by tilda). This is given by

$$
\frac{\partial L}{\partial \tilde{v}^{i}}=E\left(g_{i}+\frac{v_{i}}{\sqrt{h}}\right) .
$$


Since the vectors $g_{i}$ and $v_{i}$ are defined in a space with the metric $\gamma_{i j}$, we can identify the right hand side of the Eq.(30) as a vector in the same 3 -space. Let us call it the momentum 3 -vector

$$
p_{i} \equiv E\left(g_{i}+\frac{v_{i}}{\sqrt{h}}\right) .
$$

It can also be verified that $H=\frac{\partial L}{\partial \tilde{v}^{i}} \tilde{v}^{i}-L \equiv p_{i} \tilde{v}^{i}-L=E$, which is a constant along the trajectory of a particle as stated in Eq.(27). Hence, it is possible to introduce Maupertuis principle $\delta \int_{\vec{x}_{1}}^{\vec{x}_{2}} p_{i} \tilde{v}^{i} d t=0$. Assuming further that the spatial part of the metric could be written in an isotropic form $d l=d l_{E} / \Phi, d l_{E}=$ $\delta_{i j} d x^{i} d x^{j}, \gamma_{i j}=\delta_{i j} \Phi^{-2}$, we get

$$
v^{2}=n^{2} \tilde{v}^{2} / \tilde{\beta}^{2}, n=\frac{1}{\Phi \sqrt{h}}
$$

and the Maupertuis variational principle yields, after lengthy algebra introducing the parameter $A$, the geodesic equation in the form

$$
\begin{gathered}
\frac{d^{2} \vec{r}}{d A^{2}}=\vec{\nabla}\left(\frac{n^{2} v^{2}}{2}\right)+\frac{d \vec{r}}{d A} \times(\vec{\nabla} \times \vec{g}), \\
d A=n^{-2} \tilde{\beta} d t, \frac{d \tau}{d A}=n^{2} \sqrt{h}
\end{gathered}
$$

where $\vec{g} \equiv\left(g_{i}\right)$. This Newtonian form of the geodesic equation, valid for both massless and massive particles, has been obtained under the only assumption that the spatial part of the metric could be written in an isotropic form. On eliminating $A$ from Eqs.(33) and (34), we can find the rotational contributions to the well known Schwarzschild orbits for light and planets [36]. The geodesic Eqs.(33),(34) are valid only under the assumption of small test body approximation (back reaction ignored, as stated in Sec.II, before Eq.(8))

Eq.(33) admits an immediate interpretation, albeit in optical parlance, as describing the motion of a particle in a "potential" $(-1 / 2) n^{2} v^{2}$ and subjected to a "Coriolis" force $\frac{d \vec{r}}{d A} \times(\vec{\nabla} \times \vec{g})$, which would appear, for instance, in the absence of gravity $(n=1, h=1)$ in a coordinate system rotating with angular velocity $\vec{\Omega}=(1 / 2)(\vec{\nabla} \times \vec{g})$. One might look at the first term in Eq.(33) as the "gravistatic" part and the second term as the "gravimagnetic" part. However, such gravimagnetic analogy practically works out only in the weak field limit of physically interesting metrics. The reason is that the coordinate transformation needed to achieve the spatially isotropic form in general entails use of implicit functions. To avoid this complication, one focuses attention only to large distances on the equatorial plane (see Sec.V below). It would be interesting to compare and contrast the full optical analogy equations with the general relativistic mechanical equations developed in Ref.[39]. The gravimagnetic Eqs.(33), (34) do yield the known equations in the weak field, slow velocity limit [40]. In 
CS modified gravity, the gravimagnetic analogy was first applied by Alexander and Yunes [28] followed by Smith et al [29].

If one considers that gravity is given by the nonrelativistic, Newtonian law $\tilde{v}^{2}=\frac{2 M}{r}$ in an otherwise flat space $(n=1, h=1)$ so that $d A=d \tau$, then one has from Eq.(33), the component of radial acceleration

$$
\frac{d \tilde{v}_{\perp}}{d \tau}=-g+2|\overrightarrow{\tilde{v}} \times \vec{\Omega}|,
$$

which leads to

$$
\Delta \phi_{S R}=[-g+2|\overrightarrow{\tilde{v}} \times \vec{\Omega}|]\left(\frac{\tilde{p} A}{\hbar \tilde{v}^{2}}\right)
$$

where

$$
-g=\frac{1}{2} \frac{d}{d r}\left(\tilde{v}^{2}\right)=-\frac{M}{r^{2}} .
$$

One can see how beautifully the effects of gravity and rotation, otherwise considered as separate components in the literature [cases (ii) and (iii) in Sec.III], are synthesized into a single equation. We are now ready to apply Alsing Eqs.(33), (34) for calculating the phase shift in CS gravity.

\section{Phase shift in CS gravity}

To apply Eqs.(33), (34) we need to cast the KMT metric (6) in a spatially isotropic form. In general this is not possible, so for practical reasons, we restrict ourselves to equatorial plane $(\theta=\pi / 2)$ and to the weak field regime of the rotating source, $r \gg 2 M$. Neglecting $\omega^{2}$, we have

$$
\begin{gathered}
d s^{2}=-h(r)\left[d t+\frac{\omega r^{2}}{h(r)} d \phi\right]^{2}+\left(1-\frac{2 M}{r}\right)^{-1} d r^{2}+r^{2} d \phi^{2}, \\
h(r)=1-\frac{2 M}{r} \\
g_{\phi}=-\frac{\omega(r) r^{2}}{h(r)} .
\end{gathered}
$$

To achieve the isotropic form, we adopt isotropic radial variable $\rho$ via $\Phi=\frac{\rho}{r}$ and $\frac{d \rho}{\rho}=(1+M / r)(d r / r)$, and obtain $\rho=r e^{-\frac{M}{r}}$, which yields $r=\rho+M$, to lowest order in $\frac{M}{r}$. Thus, in all, we have the expressions with $n$ given by Eq.(32):

$$
\begin{gathered}
\Phi(\rho) \simeq 1-\frac{M}{\rho}, h(\rho) \simeq 1-\frac{2 M}{\rho}, \\
n(\rho) \simeq 1+\frac{2 M}{\rho}, g_{\phi} \simeq-\left(1+\frac{2 M}{\rho}\right)(\rho+M)^{2} \omega(\rho+M),
\end{gathered}
$$

and the metric (38) for $\frac{M}{\rho} \ll 1$ takes the form

$$
d s^{2}=-\left(1-\frac{2 M}{\rho}\right)\left[d t+g_{\phi} d \phi\right]^{2}+\left(1+\frac{2 M}{\rho}\right)\left[d \rho^{2}+\rho^{2} d \phi^{2}\right] .
$$


With these at hand, and using Eq.(33), we can rewrite the radial and cross radial components of "acceleration" as follows:

$$
\begin{gathered}
\frac{d^{2} \rho}{d A^{2}}-\rho\left(\frac{d \phi}{d A}\right)^{2}=\frac{d}{d \rho}\left(\frac{n^{2} v^{2}}{2}\right)+\left|2 \frac{d \vec{\rho}}{d A} \times \vec{\Omega}\right|, \\
\rho \frac{d^{2} \phi}{d A^{2}}+2 \frac{d \rho}{d A} \frac{d \phi}{d A}=-\frac{1}{\rho} \frac{d \rho}{d A} \frac{d g_{\phi}}{d \rho}
\end{gathered}
$$

where $\vec{\Omega} \equiv \frac{1}{2}(\vec{\nabla} \times \vec{g})$ is the angular velocity of rotation of the gravitating mass $M$. The last term in Eq.(44) represents "Coriolis force". The first integral of Eq.(45) is

$$
\frac{d}{d A}\left(\rho^{2} \frac{d \phi}{d A}+g_{\phi}\right)=0 \Rightarrow \rho^{2} \frac{d \phi}{d A}+g_{\phi} \equiv \ell
$$

where $\ell$ is an arbitrary constant of integration, which may be interpreted as the conservation of total "angular momentum". Thus

$$
\frac{d \phi}{d A}=\frac{\ell-g_{\phi}}{\rho^{2}}
$$

So far we have been talking in the language of optical analogue ("..") of mechanical quantities. Let us now translate them into mechanical quantities proper, quantities that are actually measured in a gravity field. Thus we calculate the radial acceleration $\frac{d v_{\perp}}{d \tau}$ as follows. Converting $A \rightarrow \tau$ by means of Eq.(34), we can rewrite Eq.(44) as

$$
\begin{gathered}
\frac{d v_{\perp}}{d \tau} \equiv \frac{d^{2} \rho}{d \tau^{2}}-\rho\left(\frac{d \phi}{d \tau}\right)^{2} \\
=\left(\frac{1}{n^{4} h}\right)\left[\frac{d}{d \rho}\left(\frac{n^{2} v^{2}}{2}\right)+\left(n^{2} \sqrt{h}\right)\left(2 \Omega_{n} v\right)\right]
\end{gathered}
$$

where we have used in Eq.(44) the following: $\left|2 \frac{d \vec{\rho}}{d A} \times \vec{\Omega}\right|=2\left(\frac{d \rho}{d \tau} \frac{d \tau}{d A}\right) \Omega_{n}$ together with $\frac{d \rho}{d \tau}=v, \frac{d \tau}{d A}=n^{2} \sqrt{h}, \Omega_{n}$ being the angular velocity of rotation of $M$ normal to the plane of the interferometer. We now need to calculate $\widetilde{\beta}$. Using Eqs.(29), (34) and Eq.(47) we find

$$
\tilde{\beta}=1-g_{\phi} \frac{d \phi}{d t}=1-g_{\phi} \frac{d \phi}{d A} \frac{d A}{d t}=1-g_{\phi}\left[\frac{\ell-g_{\phi}}{n^{2} \rho^{2}}\right] \tilde{\beta}
$$

Since we disregard terms of the order of $g_{\phi}^{2}$, being of the order of $\omega^{2}$, we end up with

$$
\tilde{\beta}^{-1} \simeq\left[1+\frac{g_{\phi} \ell}{n^{2} \rho^{2}}\right] .
$$


Then noting that $\tilde{v}^{2}=2 M / r \simeq 2 M / \rho$, and using $n$ from Eqs.(42) and $\tilde{\beta}$ from Eq.(51), we can finally express $v$ of Eq.(32) as

$$
v=\frac{n \widetilde{v}}{\widetilde{\beta}}=\left(1+\frac{2 M}{\rho}\right) \sqrt{\frac{2 M}{\rho}}\left[1+\frac{g_{\phi} \ell}{n^{2} \rho^{2}}\right] .
$$

On the other hand, we have the conserved proper energy and momentum for a test particle of rest mass $m$ as follows [cf. Eq.(27)]

$$
\begin{gathered}
E=\frac{m \sqrt{h}}{\sqrt{1-v^{2}}}=\widetilde{E} \sqrt{h} \\
p=\frac{m v}{\sqrt{1-v^{2}}} \Phi^{-1}=\widetilde{p} \Phi^{-1}
\end{gathered}
$$

such that the mass-shell condition may be written in a special relativistic form

$$
m^{2}=h \widetilde{E}^{2}-\Phi^{-2} \widetilde{p}^{2}=E^{2}-p^{2} .
$$

(Note that, we could obtain, alternatively, the refractive index $n$ by putting $m=0$ so that $c=\frac{\widetilde{p}}{E}=c_{0} \Phi \sqrt{h}=c_{0} n^{-1}$.) Using Eq.(54), we can express $v^{2}$ in terms of $p$ and $\Phi$, and further introducing the quantum relation $\widetilde{p}=\hbar \widetilde{\kappa}$, we get the curved space corrected Newtonian gravity term, $\Delta \widetilde{\phi}_{g} \rightarrow \Delta \phi_{g}$ :

$$
\begin{aligned}
\Delta \phi_{g} & =-g \frac{p A}{\hbar v^{2}} \equiv-A g\left(\frac{m^{2} c_{0}^{2}+p^{2} \Phi^{2}}{\hbar c_{0}^{2} p \Phi^{2}}\right) \\
& =-g\left(\frac{A m^{2}}{\hbar^{2} \widetilde{\kappa}}\right) \Phi^{-2}-\frac{g A \widetilde{\kappa}}{c_{0}^{2}} \\
& \simeq-g\left(\frac{A m^{2}}{\hbar^{2} \widetilde{\kappa}}\right)\left(1+\frac{2 M}{\rho}\right)-\frac{g A \widetilde{\kappa}}{c_{0}^{2}}
\end{aligned}
$$

An interesting observation is that the relativistic second term is completely unaffected by gravity. Symbolically, we write the phase shift in CS gravity as

$$
\begin{aligned}
\Delta \phi_{C S G} & =\left(\frac{d v_{\perp}}{d \tau}\right) \times\left(\frac{p A}{\hbar v^{2}}\right) \\
& =\left(\frac{1}{n^{4} h}\right)\left[\frac{d}{d \rho}\left(\frac{n^{2} v^{2}}{2}\right)+\left(n^{2} \sqrt{h}\right)\left(2 \Omega_{n} v\right)\right] \times\left(\frac{p A}{\hbar v^{2}}\right) \\
& =\Delta \phi_{1}+\Delta \phi_{2},
\end{aligned}
$$

where $\Delta \phi_{1}$ and $\Delta \phi_{2}$ are gravitational and Coriolis contributions respectively. This is the exact expression that contains terms of all orders in $\left(\frac{1}{\rho}\right)$ and higher. All we have to do now is to collect the expressions for $\Phi, h, n, g_{\varphi}, v$ and $\frac{d v_{\perp}}{d \tau}$ from Eqs. (41), (42), (49) and (52), insert them in Eq.(60), expand and find contributions to lowest order in $\left(\frac{1}{\rho}\right)$.

\section{Lowest order terms}


To extract ostensibly first order terms, we ignore second and higher order terms in $M, C_{1}, C_{2}$ together with their various products. Then the shift $\Delta \phi_{1}$ to lowest order is as follows:

$$
\begin{aligned}
\Delta \phi_{1} & =\left(\frac{1}{n^{4} h}\right)\left(\frac{p A}{\hbar v^{2}}\right) \frac{d}{d \rho}\left(\frac{n^{2} v^{2}}{2}\right) \\
& \simeq\left[1+\ell\left\{\frac{4 C_{2}}{\rho}+\frac{6 C_{1}}{\rho^{2}}\right\}+O\left(\epsilon^{2}\right)\right]\left(\Delta \phi_{g}\right)
\end{aligned}
$$

We can see how the term $\Delta \phi_{1}$, which represents only $\Delta \widetilde{\phi}_{g}$ in flat space, is modified by the quantities $C_{1}, C_{2}$ and $\frac{M}{\rho}$.

To calculate the Coriolis effect, we introduce the quantum relation $\widetilde{E}=\hbar \widetilde{\omega}$ so that

$$
\begin{aligned}
\left(\frac{p A}{\hbar v^{2}}\right) & =\frac{A E \Phi^{-1}}{\hbar v \sqrt{h}}=\frac{A \widetilde{\omega} \Phi^{-1}}{v} \\
& \Rightarrow\left(2 \Omega_{n} v\right)\left(\frac{p A}{\hbar v^{2}}\right)=2 \widetilde{\omega} \Omega_{n} A \Phi^{-1} .
\end{aligned}
$$

Thus we get from Eqs.(60) and (64)

$$
\Delta \phi_{2}=\left[\left(n^{2} \sqrt{h}\right)^{-1}\right] \Phi^{-1}\left(2 \widetilde{\omega} \Omega_{n} A\right) \equiv\left(1+\frac{4 M}{\rho}\right)\left(2 \widetilde{\omega} \Omega_{n} A\right) .
$$

Using the dispersion relation from Eq.(55)

$$
\widetilde{\omega}^{2}=\frac{m^{2}}{\hbar^{2}}\left[\frac{1}{h(\rho)}+\frac{\Phi^{-2}}{h(\rho)}\left(\frac{\hbar^{2} \widetilde{\kappa}^{2}}{m^{2}}\right)\right]
$$

in $2 \widetilde{\omega} \Omega_{n} A$, we explicitly obtain the gravity corrected Coriolis term, $\Delta \widetilde{\phi}_{c o r} \rightarrow$ $\Delta \phi_{\text {cor }}$ :

$$
\begin{aligned}
\Delta \phi_{c o r} & =2 \widetilde{\omega} \Omega_{n} A \\
& \simeq\left(1+\frac{M}{\rho}\right)\left[\frac{2 A \Omega_{n} m}{\hbar}\right]+\left(1+\frac{3 M}{\rho}\right)\left[\frac{\hbar \widetilde{\kappa}^{2} A \Omega_{n}}{m c_{0}^{2}}\right] .
\end{aligned}
$$

Thus we have, from Eq.(65),

$$
\Delta \phi_{2}=\left(1+\frac{4 M}{\rho}\right)\left(\Delta \phi_{c o r}\right)
$$

Recombining it with Eq.(62), we get

$$
\Delta \phi_{C S G} \simeq\left[1+\ell\left\{\frac{4 C_{2}}{\rho}+\frac{6 C_{1}}{\rho^{2}}\right\}\right]\left(\Delta \phi_{g}\right)+\left(1+\frac{4 M}{\rho}\right)\left(\Delta \phi_{c o r}\right)
$$


Recall that $C_{1}$ has the dimension of length, $C_{2}$ is dimensionless and $\ell$ has the dimension of length, hence the CS effect $\ell\left\{\frac{4 C_{2}}{\rho}+\frac{6 C_{1}}{\rho^{2}}\right\}$ is dimensionless, as should be the case. Eq.(69) is the main result of our paper.

As we see, the effect is coupled with $\ell$ which is a sum of "angular momentum" $\rho^{2} \frac{d \phi}{d A}$ of particles and $g_{\phi}$ of the source. The interferometer plane is usually placed at two vertical orientations so that the angular momentum of particles moving in the plane is practically zero. We are then left with only the source influence $g_{\phi}$, which, when multiplied by $C$, becomes an effect in $O\left(\epsilon^{2}\right)$. Nevertheless, a number of observations can be made. It is immediate that in the limit of flat space $\left(M=0, C_{1}, C_{2}=0\right)$, we recover the individual special relativistic components $\Delta \widetilde{\phi}_{g}$ and $\Delta \widetilde{\phi}_{c o r}$ derived by Anandan [14]. This means, in this limit, $\Delta \phi_{C S G}=\Delta \phi_{S R}$. If $C_{1}, C_{2}=0$, but $M \neq 0$, we recover the influence of Schwarzschild gravity embodied in the factors of $\left(\frac{M}{\rho}\right)$, so that $\Delta \phi_{C S G}=\Delta \phi_{S c h}$. It is a new result by itself. If the black hole mass $M=0$ but $C_{1}, C_{2} \neq 0$, we are left with the effect of a mere rotating coordinate system, which is of no physical interest. The remarkable result is that the parameters $C_{1}, C_{2}$ do not affect the Coriolis term at all, while it modifies only the pure gravity term $\Delta \phi_{g}$. This is because $v$, the carrier of $C_{1}, C_{2}$ via $g_{\phi}$, cancels out as shown in Eq.(64).

Since the CS component of the phase shift depends crucially on the factor $\ell$, let us analyze what different values of it means. Although it is an optical constant, we see from Eq.(46) that it is also constant in proper time, $\frac{d \ell}{d \tau}=0$, because $\frac{d \tau}{d A} \neq 0$. Note further that $\ell$ consists of two parts: A part $\rho^{2} \frac{d \phi}{d A}$, proportional to the usual particle orbital angular momentum, and a part $g_{\phi}$ proportional to the angular momentum of the black hole itself. If $\ell=0$, the CS effect vanishes altogether. This is the case when a radially freely falling particle acquires an angular velocity $\frac{d \phi}{d t} \propto \frac{g_{\phi}}{\rho^{2}}$ near the source in the same sense as the rotation of the black hole, exactly similar to what happens in the Lense-Thirring drag [41]. On the other hand, if $\ell=g_{\phi}$, then $\rho^{2} \frac{d \phi}{d A}=0 \Rightarrow \frac{d \phi}{d t}=0$, that is, the particle is at rest at constant $(r, \theta)$ relative to distant stars, and to remain at rest it has to swim against the spacetime drag. In this case, we see from Eq.(51) that there can be no $O(\epsilon)$ effect because the lowest order CS component would go like $g_{\phi} \times C \sim O\left(\epsilon^{2}\right)$, which we have agreed to ignore. Locally nonrotating [42] particles will have an angular velocity $\frac{d \phi}{d t}=-g_{t \phi} / g_{\phi \phi}=\frac{\omega(r)}{\sin ^{2} \theta}$ but again the order of the effect would go like $\omega \times C \sim O\left(\epsilon^{2}\right)$. Thus the CS effect can appear only in $O\left(\epsilon^{2}\right)$. This is a bit unfortunate. The spin precession effect, as discussed by KMAT, seems better suited to study the CS effect.

\section{Summary}

As shown by KMAT [33], the constant parameter $C_{2}$ in their solution is the observed (roughly constant) tangential velocity of particles moving in equatorial circular orbits in the galactic halo. Our aim here was to theoretically investigate the influence of such CS parameters on the quantum phase shift in thermal neutron interferometry. To achieve this goal, several steps were needed. We first established the stability of circular orbits in the CS theory, which is essential 
for the physical viability of the model itself. The second step was to preview Anandan's arguments. In the third step, we familiarized the readers with the notion of curved spacetime optical-mechanical analogy, especially the reformulation of geodesic equations developed by Alsing [36]. (We relax the assumption that KMT solution (6) replaces that of Kerr but there are indications that the CS modification is a non-trivial perturbation of Kerr geometry [35]). In the final step, we applied these equations in the linear aproximation to obtain the effects on the phase shift contributed by inertial forces, special relativity, general relativity and the CS modified theory.

Eq.(69) shows that, to leading order, the CS constants influence only the pure gravity term $\Delta \phi_{g}$. However, the constant $C_{1}$ is observationally clueless yet, but $C_{2}$ already has a solid observable meaning. We can set $C_{1}=0$ without any loss of rigor, since $\omega(r)$ of Eq.(7) would still satisfy the core CS equation $\left[r(r-2 M) \omega^{\prime \prime}+4(r-2 M) \omega^{\prime}+2 \omega=0\right]$. Stability of circular orbits is not hampered when $C_{1}=0$. Hence, leaving aside relativistic terms $O\left(c_{0}^{-2}\right)$, we have $\Delta \phi_{C S G} \simeq[1+(\alpha+\beta)+\alpha \beta]\left(\Delta \widetilde{\phi}_{g}\right)+(1+\gamma)\left(\Delta \widetilde{\phi}_{\text {cor. }}\right)$, where $\alpha=\frac{4 \ell C_{2}}{\rho}$, $\beta=\frac{2 M}{\rho}, \gamma=\frac{5 M}{\rho}$, representing respectively the CS and Schwarzschild influences on the observed flat space effects of Newtonian gravity and inertial forces.

One might argue that the mass $M$ in question is actually that of the central galactic bulge and any possibility of interferometry is out of question. We wish to argue differently. Observationally, the frequency shifts in the HI radiation show that, in the halo region, $C_{2}=v^{\varphi} / c_{0}$ is nearly constant at a value $7 \times 10^{-4}$ [43]. We may take this value of $C_{2}$ as permanently fixed (up to observational uncertainty), like cosmological constant, and treat the KMT solution as completely determined like Schwarzschild-de Sitter solution. Then we can apply the solution to study the nonlocal effect of this constant on the measurement of phase shifts for any rotating source, including Earth. To lowest order, and assuming $\omega \simeq \frac{C_{2}}{\rho+M}$ in Eq.(42), we see that $\alpha=\frac{4 g_{\phi} C_{2}}{\rho}=4 C_{2}^{2}\left(1+\frac{3 M}{\rho}\right) \sim 2 \times 10^{-6}$. The Schwarzschild $(\beta, \gamma)$ corrections are of the order of $\frac{M}{\rho} \sim 10^{-9}$ on Earth. Thus the current accuracy level measuring $\Delta \phi_{g}$ must be augmented by $10^{6}$ times for CS effect and $10^{9}$ times for the Schwarzschild effect. Whatever be the experimental scenario, our final result, Eq.(69), provides us with a clear view of the various effects within a single theoretical framework. It would be interesting to do analogous calculations for Dirac particles. But it would be highly nontrivial since the Dirac Lagrangian density would have to be coupled to the CS field, through the covariant derivative. Perhaps a starting point could be the analysis of Alexander and Yunes [31] but extending this to spinning particles would be very hard.

Finally we would like to summarize the problematic characteristics of the KMT solution it has inherited from the non-dynamical framework. First, the solution is not a well accepted replacement of slow rotation limit of the Kerr solution. The KMT solution is a very special line element that holds only for a particular choice of the CS coupling field. Unlike in the Kerr case, there is no Robinson's theorem [44] for rotating sources in CS gravity that could suggest that this solution is unique. Indeed, Yunes and Pretorius [35] have found 
a family of new stationary axisymmetric solutions in the non-dynamical theory, which shows that solutions in this theory can not be unique. From this standpoint, the far-field solutions of Alexander and Yunes, and Smith et al are equally valid. Second, the KMT solution is incompatible with the dynamical formulation of CS modified gravity. This is because the energy contained in the KMT CS scalar is infinite. The Yunes-Pretorius solution bypasses the problem with the stress tensor and is compatible with dynamical theory. Third, the non-dynamical framework (upon which both the KMT solution [34] and the far-field solutions of Alexander and Yunes [27, 28] are based) is problematic for Schwarzschild black hole perturbation theory, as shown by Yunes and Sopuerta [32]. In this work, the authors showed that there cannot be single-parity perturbations in non-dynamical CS modified gravity, which suggests that when a small object falls head-on into a supermassive black hole, the latter cannot be perturbed to linear order. This obstacle is partially resolved in the dynamical theory. Fourth, the non-dynamical framework is also problematic from the theoretical standpoint. The main objection comes from the external, purely ad hoc prescription of the CS coupling field, which is not motivated by considerations from within the theory. Moreover, the non-dynamical framework also contains an additional constraint (the Pontryagin constraint), which suggests that the modified field equations are over-constrained. Indeed, this constraint affects the black hole perturbation theory (Yunes and Sopuerta [32]), and the search for exact solutions that could represent spinning black holes (Grumiller and Yunes [30]). If this is the case, then one might conclude that the non-dynamical framework can only have an ill-posed initial value problem [32].

\section{Figure captions:}

Fig,1 The height represents $V^{\prime \prime}$ for small values of $C_{1} \neq 0, C_{2} \neq 0$ along $\mathrm{x}$ and $\mathrm{y}$ axes respectively. The radius is arbitrary but fixed at any $r=R$. Negative values of $V^{\prime \prime}$ indicate stability.

Fig.2. The de Broglie wavelets originating at $A$ and interfering at $B$, one travelling along $A D B$ and the other along $A C B$, the plane $A D B C$ being rotated about the horizontal axis $A O$ (Ref.[14]).

\section{Acknowledgments:}

This paper is dedicated to Professor Swapan K. Ghosal on his $62^{\text {nd }}$ birthday. We are deeply indebted to Professor Alexander I. Filippov for many stimulating discussions and to Guzel N. Kutdusova, Denis V. Kondratiev, Aydar R. Bikmetov and Rustam R. Fazlaev for their interest and useful assistance. We sincerely thank the anonymous referee for many incisive and informative comments. KKN acknowledges warm hospitality at BSPU and SSPA.

\section{References}

[1] The literature is too vast. However, for a review on the state of research in quantum gravity, see: C. Rovelli, Living Reviews in Relativity, vol.1 (http://www.livingreviews.org/Article). 
[2] R. Colella, A.W. Overhauser, and S.A. Werner, Phys. Rev. Lett. 34, 1472 (1975); A.W. Overhauser and R. Colella, Phys. Rev. Lett. 33, 1237 (1974); D. Greenberger and A.W. Overhauser, Rev. Mod. Phys. 51, 43 (1979).

[3] S.A. Werner, J.-L. Staudenmann, and R. Colella, Phys. Rev. Lett. 42, 1103 (1979); T.L. Gustavson, P. Bouyer, and M.A. Kasevich, Phys. Rev. Lett. 78, 2046 (1997).

[4] Lorne A. Page, Phys. Rev. Lett. 35, 543 (1975).

[5] T. Padmanabhan, T.R. Seshadri, and T.P. Singh, Int. J. Mod. Phys. A 1, 491 (1986); V. Daftardar and N. Dadhich, Int. J. Mod. Phys. A 2, 731 (1987); K.K. Nandi, A. Islam, and J. Evans, Int. J. Mod. Phys. A 12, 3171 (1997).

[6] Abhay Ashtekar and Anne Magnon, J. Math. Phys. 16, 342 (1975); N. Fornengo, C. Giunti, C.W. Kim, and J. Song, Phys. Rev. D 56, 1895 (1997); C. Giunti, C.W. Kim, and U.W. Lee, Phys. Rev. D 44, 3635 (1991); S. Capozziello and G. Lambiase, Mod. Phys. Lett. A 14, 2193 (1999).

[7] D.V. Ahluwalia and C. Burgard, Phys. Rev. D 57, 4724 (1998); D.V. Ahluwalia and C. Burgard, Gen. Rel. Grav. 28, 1161 (1996); J. Wudka, Mod. Phys. Lett. A 6, 3291 (1991).

[8] T. Bhattacharya, S. Habib, and E. Mottola, Phys. Rev. D 59, 067301 (1999).

[9] M. Gasperini, Phys. Rev. D 39, 3606 (1989); A. Halprin and C.N. Leung, Phys. Rev. Lett. 67, 1833 (1991).

[10] L. Stodolsky, Gen. Rel. Grav. 11, 391 (1979).

[11] P.M. Alsing, J.C. Evans, and K.K. Nandi, Gen. Rel. Grav. 33, 1459 (2001).

[12] Y. Fukuda et al., Phys. Rev. Lett. 82, 1810 (1998).

[13] G. Amelino-Camelia, J. Ellis, N.E. Mavromatos, D.V. Nanopoulos, and S. Sarkar, Nature (London), 393, 763 (1998).

[14] J. Anandan, Phys. Rev. D 15, 1448 (1977); J. Anandan, Nuovo Cim. A 53, 221 (1979).

[15] K.K. Nandi and A. Islam, Am. J. Phys. 63, 251 (1995)

[16] J. Evans, A. Islam, and K.K. Nandi, Am. J. Phys. 64, 1404 (1996)

[17] J. Evans, A. Islam, and K.K. Nandi, Gen. Rel. Grav. 28, 413 (1996)

[18] J. Evans, P.M. Alsing, S. Giorgetti, and K.K. Nandi, Am. J. Phys. 69, 1103 (2001).

[19] J. Evans and M. Rosenquist, Am. J. Phys. 54, 876 (1986).

[20] K.K. Nandi and Y.Z. Zhang, Phys. Rev. D 66, 063005 (2002).

[21] M.B. Green and J.H. Schwarz, Phys. Lett. 149 B, 117 (1984); A. Sen, Phys. Rev. Lett. 69, 1006 (1992).

[22] J. Polchinski, String theory, Cambridge University Press, Cambridge (1998)

[23] S.H.S. Alexander, M.E. Peshkin, and M.M. Sheikh-Jabbari, Phys. Rev. Lett. 96, 081301 (2006); eprint hep-th/0701139.

[24] S.H.S. Alexander and S.J. Gates Jr., J. Cosmol. Astropart. Phys. 06 (2006) 018.

[25] R. Jackiw and S.-Y. Pi, Phys. Rev. D 68, 104012 (2003). 
[26] D. Guarrera and A.J. Hariton, Phys. Rev. D 76, 044011 (2007).

[27] S.H.S. Alexander and N. Yunes, Phys. Rev. Lett. 99, 241101 (2007).

[28] S.H.S. Alexander and N. Yunes, Phys. Rev. D 75, 124022 (2007).

[29] T. L. Smith, A. L. Erickcek, R. R. Caldwell, and M. Kamionkowski, Phys. Rev. D 77, 024015 (2008).

[30] D. Grumiller and N. Yunes, Phys. Rev. D 77, 044015 (2008).

[31] S.H.S. Alexander and N. Yunes, Phys. Rev. D 77, 124040 (2008).

[32] N.Yunes and C.F. Sopuerta, Phys. Rev. D 77, 064007 (2008).

[33] K. Konno, T. Matsuyama, Y. Asano, and S. Tanda, Phys. Rev. D 78, 024037 (2008).

[34] K. Konno, T. Matsuyama, and S. Tanda, Phys. Rev. D 76, 024009 (2007).

[35] N. Yunes and F. Pretorius, eprint: gr-qc/0902.4669.

[36] P.M. Alsing, Am. J. Phys. 66, 779 (1998).

[37] M. Mathisson, Acta Phys. Polon. 6, 163 (1937); A. Papapetrou, Proc. Roy. Soc. London, A 209, 258 (1951).

[38] L.D. Landau and E.M. Lifschitz, The Classical Theory of Fields, Pergamon, New York (1975), 4th Ed.

[39] K.S. Thorne, R.H. Price, and D. MacDonald, Black Holes: The Membrane Paradigm, Yale University Press, New Haven (1986).

[40] E. Harris, Am. J. Phys. 59, 421 (1991).

[41] In the Kerr case, $g_{\phi}=-\frac{2 M a}{\rho}$. Solving for $\frac{d \phi}{d t}$, using the weak field approximation, $\rho=r-M$, it follows that $\ell=0$ leads to $\rho^{2} \frac{d \phi}{d t}=\frac{2 M a}{\rho}$. It means that the test particle coming straight in from infinity acquires an angular velocity $\frac{2 M a}{\rho^{3}}$ in the same sense as the rotation of $M$. This is essentially the Lense-Thirring frame dragging effect.

[42] C.W. Misner, K.S. Thorne and J.A. Wheeler, Gravitation, Freeman, San Francisco (1973), p. 895.

[43] J.J. Binney and S. Tremaine, Galactic Dynamics, Princeton University Press, New Jersey (1987); A. Borriello and P. Salucci, Mon. Not. R. Astron. Soc. 323, 285 (2001); M. Persic, P. Salucci, and F. Stel, Mon. Not. R. Astron. Soc. 281, 27 (1996).

[44] D.C. Robinson, Phys. Rev. Lett. 34, 905 (1975). 